Nasenventil bei obstruktiver Schlafapnoe

\title{
Gute Compliance, aber schlechte Wirkung
}

Rosenthal L et al. A multicenter, prospective study of a novel nasal EPAP device

in the treatment of obstructive sleep apnea: efficacy and 30-day adherence.

J Clin Sleep Med. 2009;5:532-537

\section{Hintergrund}

Die gängigen Therapien für Patienten mit obstruktivem Schlafapnoesyndrom (OSAS) wie die kontinuierliche positive Überdrucktherapie (CPAP) oder das Tragen einer Protrusionsschiene werden entweder schlecht akzeptiert oder nicht regelmäßig durchgeführt. Es besteht deshalb ein Bedarf für weitere therapeutische Optionen.

\section{Fragestellung}

Die „Provent Sleep Apnea Therapy“ (Ventus Medical) besteht aus einem Einwegeventil, das in die Nasenöffnungen eingeführt wird. Das Ventil öffnet sich beim Einatmen und schließt sich beim Ausatmen, wobei eine kleine Öffnung bleibt. Dadurch entsteht während der Exspirationsphase ein positiver Atemwegsdruck. Erste Untersuchungen an 34 Patienten ergaben, dass unter Verwendung dieses Ventils der Apopnoe-Hypopnoe-Index (AHI) und das Schnarchen reduziert werden. Unklar war bis jetzt, ob und in welchem Ausmaß sich der AHI bei verschiedenen exspiratorischen Widerständen verändert. Rosenthal und Kollegen untersuchten diese Frage.

\section{Methodik}

In einer randomisierten Multicenterstudie wurde die Wirkung verschiedener exspiratorischer Widerstände eines Ausatemventils für die Nase an 28 Patienten mit OSAS (AHI > 5/h) verglichen.

\section{Ergebnisse}

Der mittlere AHI betrug während der Kontrollnacht 24,5/h. Unter den exspiratorischen Widerständen 50, 80 und 110 $\mathrm{cmH}_{2} \mathrm{Osek} / \mathrm{l}$ fiel der AHI auf 13,6, 12,5 bzw. 14,4/h (Durchschnitt: 13,5/h).

Für die nächsten 30 Tage bekamen die Patienten das Ausatemventil mit nach
Hause. Dort sollten sie den individuell günstigsten Widerstand anwenden. In der Kontrollnacht nach 30 Tagen lag der AHI bei 15,5/h. Der prozentuale Anteil des Schnarchens während der Gesamtschlafzeit fiel nach 30 Tagen von 27,5 auf 14,6\%. Der Sauerstoffdesaturationsindex und die minimale Sauerstoffsättigung veränderten sich nicht signifikant. Der Epworth Sleepiness Score (ESS) als Maß für die Schläfrigkeit fiel von 8,7 auf 6,9.

Die Patienten berichteten über eine durchschnittliche Nutzung des Nasenventils während 94,4\% aller Nächte. Die beobachteten unerwünschten Ereignisse wie Ohrenschmerzen oder rauer Hals standen nicht in Zusammenhang mit dem Ausatemventil.

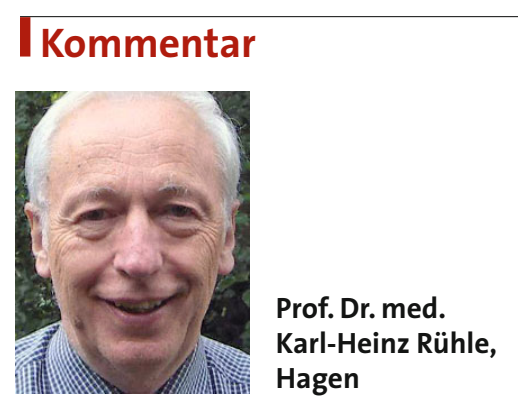

Für den Erfolg dieser Therapie bezüglich des $\mathrm{AHI}$ kommen mehrere Mechanismen infrage. Bei Beginn der Inspiration könnte noch ein positiver Atemwegsdruck vorliegen, sofern die Exspirationszeit durch den erhöhten Ausatmungswiderstand stark verlängert wird, sodass die Kollapsneigung zu Beginn der Inspiration verringert ist. Manche Patienten aktivieren allerdings während der passiven Ausatmung die exspiratorische Atemmuskulatur, sodass die Ausatmungszeit durch den erhöhten Widerstand nicht verlängert wird. Während der Inspiration kommt es relativ schnell zu einer Aktivierung der dilatierenden pharyn- gealen Muskulatur, sodass sich die Kollapsneigung des Oropharynx verringert.

Ein erhöhter endexspiratorischer Druck erhöht das Lungenvolumen, sodass die oberen Atemwege durch Traktion vorgespannt und damit stabilisiert werden. Eine Erhöhung des Lungenvolumens hängt von der Lungen- und Thoraxwandcompliance $a b$. Dies dürfte unter anderem der Grund sein, weshalb nicht alle Patienten ansprachen.

Eine Erhöhung des Atemwegsdrucks ist nur möglich, wenn der Mund geschlossen ist. Bei erhöhtem nasalem Widerstand wird häufig der Mund geöffnet. Unklar bleibt, bei welchem nasalen Widerstand ein Patient von der Nasen- zur Mundatmung wechselt.

Eine hohe Effektivität der Therapie muss bezweifelt werden. Nur bei zehn von $24 \mathrm{~Pa}$ tienten mit einem $\mathrm{AHI}>10 / \mathrm{h}$ fiel der $\mathrm{AHI}$ auf $<10 / \mathrm{h}$. Sowohl der Desaturationsindex als auch die Schlafqualität veränderten sich nicht. Die Autoren betonen, dass die Ergebnisse einen variablen und zum Teil inkonsistenten Nutzen der Therapie reflektieren.

Ein weitere Alternative ist die transnasale Insufflation (TNI), bei der mit hoher Flussrate $(20-30 \mathrm{l} / \mathrm{min})$ warme und angefeuchtete Luft zugeführt wird. Mit der offenen Nasenkanüle, die den Patienten relativ gering beeinträchtigt, gelingt es, einen kontinuierlichen, allerdings nur geringen Überdruck in den oberen Atemwegen zu erzielen und die Kollapsneigung zu reduzieren. In einer Studie fiel der Respiratory Disturbance Index (RDI) unter TNI von 22,6 auf 17,2 Ereignisse/Stunde [1]. Eine therapeutisch relevante Reduktion auf < 10 Ereignisse/Stunde fand sich bei $27 \%$ der Patienten mit OSAS.

\section{Fazit}

Bei der CPAP-Therapie ist eine hohe Erfolgsrate mit dem Nachteil einer geringeren Nutzungszeit verbunden. Dagegen findet sich unter der Therapie mit dem nasalen Ausatemventil eine geringere Ansprechrate, aber eine hohe Anwendungsrate und Nutzungszeit. Weitere Untersuchungen sind erforderlich, um den Stellenwert dieser alternativen Therapieformen richtig einordnen zu können. Die besten Ergebnisse werden wahrscheinlich bei leicht- bis mittelgradigem OSAS erreicht werden können. Die Therapie mit CPAP bei OSAS wird weiterhin Goldstandard bleiben.

\section{Literatur}

1. Nilius G et al. Chest 2009 (E-pub ahead of print) 